\title{
Calculations of energies of intrashell doubly excited states of beryllium-like ions
}

\author{
Z Chen and C D Lin \\ Department of Physics, Kansas State University, Manhattan, KS 66506, USA
}

Received 30 January 1989, in final form 3 May 1989

\begin{abstract}
Energy levels of doubly excited states of beryllium-like ions are calculated using the conventional configuration-interaction method. The interactions of the two outer electrons with the inert $1 s^{2}$ core electrons are represented by a model potential. The resulting energies of $1 \mathrm{~s}^{2} 3 l 3 l^{\prime}$ and $1 \mathrm{~s}^{2} 4 l 4 l^{\prime}$ doubly excited states, designated in terms of the $K, T$ and $A$ correlation quantum numbers, are used to identify the Auger electron spectra derived from the collision of multiply charged ions with atoms and molecules. The systematics of energy levels is analysed and compared with the supermultiplet structure of doubly excited states of helium-like ions.
\end{abstract}

\section{Introduction}

Doubly excited states of positive ions have been observed frequently in recent years in the collision of slow multiply charged ions with atoms or molecules. These states, where two electrons are excited from the ground-state configuration, are formed either by double electron capture processes, where two electrons are transferred to the projectile ion in a single collision (Mack et al 1989, Mack 1987, Bordenave-Montesquieu et al 1987, Mann 1987), or by transfer excitation processes, where one of the projectile electrons is excited together with the capture of an electron from the target. These doubly excited states are identified by Auger electron spectroscopy or through their radiative decays.

One major difficulty in the study of such collisions is the lack of knowledge of the energy levels of doubly excited states for most atoms and ions. Extensive calculations for the energies (and sometimes the widths) of doubly excited states have been carried out only for helium-like two-electron systems (Ho 1987, Oza 1987, Lipsky et al 1977, Lin 1989). For doubly excited states, for example, of beryllium-like ions, which have been observed in many laboratories, most of the energy levels are unknown. Without the knowledge of the energy levels of these states, it is difficult to study the collision dynamics.

In this paper our goal is to provide accurate energy levels of doubly excited states of beryllium-like ions using the conventional configuration-interaction method. These energies are to be used to assist experimentalists in identifying doubly excited states populated in ion-atom collisions. Since the two excited electrons for these states remain far away outside the closed $1 \mathrm{~s}^{2}$ core, it is most convenient to calculate the energies of doubly excited states using a model-potential approach. Such an approach has also been used recently by Martín et al (1988) in conjunction with the Feshbach method, where the two lowest ${ }^{1} \mathrm{~S}^{\mathrm{e}}$ and ${ }^{1,3} \mathrm{P}^{\mathrm{o}}$ states of the $1 \mathrm{~s}^{2} 3 l 3 l^{\prime}$ manifold have been 
calculated. In $\$ 2$ we outline the model-potential method. The results for the $1 \mathrm{~s}^{2} 3 l 3 l^{\prime}$ type doubly excited states for $Z=6,7,8$ and 10 are then presented in $\S 3.1$. In $\S 3.2$ we compare the calculated Auger energies with experimental measurements and in $\S 3.3$ we examine the spectral regularity of these states. We have also calculated the energies of $1 s^{2} 3 \ln l$ ' states for $4 \leqslant n \leqslant 7$, which are available from the authors. For $Z=10$ we also tabulate the energies for $1 \mathrm{~s}^{2} 4 l 4 l^{\prime}$ states. The concluding remarks are given in $\S 4$.

\section{The model-potential approach}

The model-potential method has been used frequently to treat atomic and ionic systems when the outer electrons are well separated from the inner electrons. In this case, the outer electrons are treated as moving in a model potential generated by the inner electrons and the nucleus. For such a model two-electron system, the Hamiltonian is (in atomic units)

$$
H=-\frac{1}{2} \nabla_{1}^{2}-\frac{1}{2} \nabla_{2}^{2}+V\left(r_{1}\right)+V\left(r_{2}\right)+1 / r_{12} .
$$

In equation (1) we do not include a dielectronic polarisation potential (Norcross and Seaton 1969), which is to give a correct asymptotic form of the potential when one of the outer electrons is far away. The effect of such a term on the energy levels is small.

Various forms of the model potential $V(r)$ have been used. Norcross and Seaton (1969) represented the ionic core by the Thomas-Fermi potential plus a polarisation term with parameters adjusted to fit the first few low-lying one-electron energies. A different form of the model potential was used by Laughlin and Victor (1972). In their approach, the screening of the core electrons was obtained by approximating the core electrons with Hartree-Fock wavefunctions. The model potential then includes shortrange terms as well as polarisation terms and the parameters in the potential are determined by fitting to the experimental energies of the one-electron ions. With the model potential thus determined, the resulting two-electron Hamiltonian is then solved for bound states as well as for scattering states. Previous applications (Stewart et al 1974) of the model-potential approach to the beryllium isoelectronic sequence have been limited to the energy levels and oscillator strengths for bound states of the $1 \mathrm{~s}^{2} 2 \mathrm{~s} n l$ type or the first few autoionising states of the $1 \mathrm{~s}^{2} 2 \mathrm{p} n l$ type. The method has also been used to calculate the electron-alkali atom collisions in the close-coupling formalism (Moore and Norcross 1974). In recent years, the model-potential approach has been used in conjunction with hyperspherical coordinates in the study of the two-valenceelectron systems (Greene 1981, Lin 1983b, Christensen-Dalsgaard 1985).

In this paper our goal is to obtain energy levels of doubly excited states of beryllium-like ions to assist experimentalists in identifying the observed electron spectra. In particular, we will present energy levels of the $1 \mathrm{~s}^{2} 3 l 3 l^{\prime}$ manifold, which have been observed in several systems in the collision between slow highly charged ions with $\mathrm{He}$ or $\mathrm{H}_{2}$. Since we are dealing with positive ions, the model potential was chosen to be simply of the form

$$
V(r)=-(Z-2) / r-2(1+\alpha r) \mathrm{e}^{-2 \alpha r} / r
$$

with only one adjustable parameter $\alpha$ to be fitted. The polarisation potential, which 
is of the form $1 / r^{4}$ at large $r$, is small in comparison with the Coulomb potential $-(Z-2) / r$ seen by the outer electron and is neglected. By solving the one-electron Schrödinger equation using the potential (2) for the first three $s$ and first three $p$ states, an optimum value of $\alpha$ was selected. In table 1 we show the energy levels of the first few low-lying states of the one-electron ions calculated with this fitting procedure and the parameter $\alpha$ for the lithium-like ions for $Z=6,7,8$ and 10 and the comparison with experimental energies (Moore 1949). We note that the error in the fitted energy is of the order of $0.05 \mathrm{eV}$ for $Z=6$ to $0.3 \mathrm{eV}$ for $Z=10$. The model potentials used in our work have the same form as those used by Martin et al (1988) where they fitted the parameter $\alpha$ so that the 2 s orbital energies of the lithium-like ions are in agreement with experiment. Their values of $\alpha$ are also listed in table 1 and the resulting $n$ s and $n \mathrm{p}(n=2-4)$ orbital energies for $\mathrm{O}^{5+}$ calculated with their potential are compared with the results we obtained.

We remark that the simple form of the potential given in (2) has an advantage in that it is also suitable for use in the scattering calculations between ions and atoms. For example, the collision between $\mathrm{C}^{4+}$ and $\mathrm{He}$ can be treated as a model two-electron

Table 1. Model-potential parameter $\alpha$ for lithium-like ions for $Z=6,7,8$ and 10 and the comparison of the calculated binding energies of $n$ s and $n \mathrm{p}(n=2-4)$ states with experiment (Moore 1949), For $\mathrm{O}^{5+}$ we also compare with the results of Martín et al (1987). All the entries are the binding energies $(\mathrm{eV})$.

\begin{tabular}{|c|c|c|c|}
\hline & Experiment & This work & Martin et al \\
\hline $\mathrm{C}^{3+}$ & & $(3.716)$ & $(3.7300)$ \\
\hline $2 s$ & 64.467 & 64.468 & \\
\hline $3 \mathrm{~s}$ & 26.934 & 26.964 & \\
\hline $4 \mathrm{~s}$ & 14.727 & 14.744 & \\
\hline $2 p$ & 56.466 & 56.546 & \\
\hline $3 p$ & 24.800 & 24.838 & \\
\hline $4 p$ & 13.863 & 13.882 & \\
\hline $\mathrm{N}^{4+}$ & & $(4.402)$ & $(4.4054)$ \\
\hline $2 s$ & 97.851 & 97.851 & \\
\hline $3 \mathrm{~s}$ & 41.320 & 41.361 & \\
\hline $4 s$ & 22.704 & 22.724 & \\
\hline $2 p$ & 89.853 & 89.921 & \\
\hline $3 p$ & 38.631 & 38.416 & \\
\hline $4 p$ & 21.612 & 21.610 & \\
\hline $\mathrm{O}^{5+}$ & & $(5.079)$ & $(5.0736)$ \\
\hline $2 s$ & 138.060 & 138.052 & 138.053 \\
\hline $3 \mathrm{~s}$ & 58.738 & 58.784 & 58.786 \\
\hline $4 \mathrm{~s}$ & 32.384 & 32.408 & 32.409 \\
\hline $2 p$ & 126.072 & 126.247 & 126.269 \\
\hline $3 p$ & 55.495 & 55.593 & 55.555 \\
\hline $4 p$ & 31.060 & 31.107 & 31.103 \\
\hline $\mathrm{Ne}^{6+}$ & & $(6.401)$ & $(6.3943)$ \\
\hline $2 s$ & 238.936 & 238.925 & \\
\hline $3 \mathrm{~s}$ & 102.673 & 102.732 & \\
\hline $4 s$ & 56.853 & 56.887 & \\
\hline $2 p$ & 222.965 & 223.300 & \\
\hline $3 p$ & 98.310 & 98.450 & \\
\hline $4 p$ & 55.050 & 55.133 & \\
\hline
\end{tabular}


system (Fritsch and Lin 1986) where the two-centre potential can be written as

$$
V\left(r_{1}, r_{2}\right)=V\left(r_{1 \mathrm{P}}\right)+V\left(r_{2 \mathrm{P}}\right)-\frac{2}{r_{1 \mathrm{~T}}}-\frac{2}{r_{2 \mathrm{~T}}}+\frac{1}{r_{12}} .
$$

In (3), $r_{i C}$ is the distance between the electron $i$ and centre $\mathrm{C}(\mathrm{P}=$ projectile, $\mathrm{T}=$ target $)$.

With the potential $V(r)$ thus constructed, the two-electron Schrödinger equation with the Hamiltonian (1) is solved by the conventional configuration-interaction $(\mathrm{Cl})$ method, which allows us to obtain the energy levels of many states for each given $L$, $S$ and parity $\pi$ in a single calculation. We have calculated all the energy levels of $3 \ln l^{\prime}(n=3-7)$ states for $L=0-4$ for $Z=6,7,8$ and 10 . For $\mathrm{Ne}^{6+}$ we also calculated the intrashell $4 l 4 l$ ' states. The two-electron basis functions were constructed from the product of hydrogenic states with an effective charge $(Z-2)$ and these basis functions are then used to diagonalise the two-electron Hamiltonian (1) to obtain eigenenergies. Such truncated cI calculations give the approximate energy levels. The autoionisation widths and the small energy shift $\Delta$ due to the interaction with the degenerate continuum states $2 \mathrm{~s} \varepsilon l^{\prime}$ and $2 \mathrm{p} \varepsilon l^{\prime}$ are not considered. More accurate calculations for individual doubly excited states can be carried out in the future but it would be very timeconsuming for all the doubly excited states considered in this paper. In our typical calculations, the basis set is of the order of 60-80 for each $L, S$ and $\pi$. In calculating the energy levels of $3 \operatorname{lnl} l^{\prime}$ states, none of the basis functions consist of orbitals with $n \leqslant 2$.

\section{Results and discussion}

\subsection{Energy levels of $1 \mathrm{~s}^{2} 3 l 3 l^{\prime}$ states}

In table 2 we show the energy levels of $1 \mathrm{~s}^{2} 3 l 3 l^{\prime}$ states calculated for $\mathrm{C}^{2+}, \mathrm{N}^{3+}, \mathrm{O}^{4+}$ and $\mathrm{Ne}^{6+}$. Energies for $1 \mathrm{~s}^{2} 3 \ln l^{\prime}$ states for $4 \leqslant n \leqslant 7$ are also calculated and can be obtained from the authors. The entries in table 2 are the binding energies $(\mathrm{eV})$ measured from the threshold of removing the two outer electrons (i.e. from the helium-like ion). Results from the recent calculations of Martín et al (1988) for the first two ${ }^{1} \mathrm{~S}^{\mathrm{e}}$ and the first two ${ }^{1,3} \mathrm{P}^{\circ}$ states are also given for comparison. We note that there is a general agreement of the energies calculated by both methods except for the lowest ${ }^{1} \mathrm{~S}^{\mathrm{e}}$ state for each ion, which amounts to $0.7 \mathrm{eV}$ for $\mathrm{C}^{2+}$ and to $1.1 \mathrm{eV}$ for $\mathrm{Ne}^{6+}$. We will discuss these discrepancies later. All the energies are given only to the second decimal point and are arranged in decreasing order, and each state is designated using the $(K, T)^{A}$ correlation quantum numbers in addition to $L, S$ and $\pi$ (Lin 1983a, 1984, 1986). Since $A=+1$ for intrashell states, the quantum number $A$ was not explicitly shown in table 2. We did not use the single-particle $3 l 3 l^{\prime}$ notation for each state since configuration mixing in general is quite large and the quantum numbers $l$ and $l^{\prime}$ are not well defined. We will address this question further in $\S 3.3$.

The difference in the energy of the lowest ${ }^{1} S^{e}$ state between the present and the calculation of Martín et al (1988) is not clear since the model potential used for each system is practically identical. We have checked the accuracy of our method by calculating the energy of the same state for the two-electron system $\mathrm{Be}^{2+}$. The energy we obtained is in good agreement with the CI calculation of Lipsky et al (1975) and of Ho (1979) to better than $0.01 \mathrm{eV}$. In Ho's calculation using the method of complex coordinate rotations the energy shift $\Delta$ was included for each state. We have also checked the energies of the first two ${ }^{1} \mathrm{~S}^{\mathrm{e}},{ }^{3} \mathrm{P}^{\circ}$ and ${ }^{1} \mathrm{P}^{\circ}$ states of $\mathrm{N}^{5+}$ in the $3 l 3 l^{\prime}$ manifold 
Table 2. Calculated binding energies $(\mathrm{eV})$ of $1 \mathrm{~s}^{2} 3 / 3 l^{\prime}$ doubly excited states of beryllium-like ions. Each state is designated in terms of the quantum numbers $(K, T)$ in addition to $L$, $S$ and $\pi$. For ${ }^{2} \mathrm{~S}^{e}$ and ${ }^{1,3} \mathrm{P}^{\mathrm{o}}$ states the results of Martín et al (1987) are also given.

\begin{tabular}{|c|c|c|c|c|}
\hline$(K, T)$ & $\mathrm{C}^{2+}$ & $\mathrm{N}^{3+}$ & $\mathrm{O}^{4+}$ & $\mathrm{Ne}^{6+}$ \\
\hline$(2,0){ }^{1} \mathrm{~S}^{\mathrm{e}}$ & $47.78(47.08)$ & $75.04(74.26)$ & $108.35(107.54)$ & $193.18(192.28)$ \\
\hline$(0,0)^{1} S^{e}$ & $41.84(41.91)$ & $67.39(67.53)$ & $99.02(99.18)$ & $180.47(180.45)$ \\
\hline$(-2,0)^{1} S^{e}$ & 36.64 & 60.46 & 90.33 & 168.26 \\
\hline$(2,0){ }^{3} \mathrm{P}^{\circ}$ & $46.13(45.84)$ & $72.95(72.64)$ & $105.83(105.53)$ & $189.80(189.53)$ \\
\hline$(0,0)^{3} \mathrm{P}^{\circ}$ & $41.09(41.18)$ & $66.42(66.54)$ & $97.83(97.96)$ & $178.83(178.99)$ \\
\hline$(1,1)^{1} \mathrm{P}^{\circ}$ & $44.32(44.14)$ & $70.63(70.43)$ & $103.38(102.82)$ & $185.97(185.80)$ \\
\hline$(-1,1)^{1} \mathrm{P}^{\circ}$ & $39.11(39.01)$ & $63.71(63.59)$ & $94.38(94.26)$ & $173.92(173.81)$ \\
\hline$(1,1)^{3} \mathrm{P}^{\mathrm{e}}$ & 43.06 & 69.05 & 101.12 & 183.47 \\
\hline$(-1,1)^{3} \mathrm{p}^{\mathrm{e}}$ & 39.33 & 64.11 & 94.96 & 174.80 \\
\hline$(2,0)^{1} \mathrm{D}^{\mathrm{e}}$ & 44.49 & 70.80 & 103.18 & 186.13 \\
\hline$(0,2)^{1} \mathrm{D}^{\mathrm{e}}$ & 41.71 & 67.24 & 98.84 & 180.21 \\
\hline$(0,0){ }^{\mathrm{I}} \mathrm{D}^{\mathrm{e}}$ & 39.21 & 63.85 & 94.56 & 174.14 \\
\hline$(1,1)^{3} \mathrm{D}^{\mathrm{e}}$ & 44.04 & 70.20 & 102.42 & 185.02 \\
\hline$(1,1){ }^{1} \mathrm{D}^{\circ}$ & 42.21 & 67.89 & 99.65 & 181.34 \\
\hline$(0,2)^{3} \mathrm{D}^{\circ}$ & 41.29 & 66.72 & 98.21 & 179.38 \\
\hline$(2,0)^{3} \mathrm{~F}^{\circ}$ & 42.35 & 68.03 & 99.77 & 181.46 \\
\hline$(1,1)^{1} \mathrm{~F}^{\circ}$ & 40.21 & 65.11 & 96.07 & 176.21 \\
\hline$(1,1)^{3} \mathrm{~F}^{\mathrm{e}}$ & 40.51 & 65.62 & 96.78 & 177.25 \\
\hline$(2,0){ }^{1} G^{e}$ & 39.91 & 64.70 & 95.54 & 175.37 \\
\hline
\end{tabular}

using our method. Our results are in good agreement with the calculations of Ho (1987). We note that the basis functions of the type $2 / n l^{\prime}$ were included in the calculation of Martín et al while these basis functions were excluded in our calculation.

\subsection{Comparison with experiments}

Extensive Auger electron spectra resulting from double electron capture from helium atoms or hydrogen molecules by multiply charged ions of $\mathrm{C}, \mathrm{N}, \mathrm{O}$ and $\mathrm{Ne}$ from the ECR source at Groningen have been reported in the thesis of Mack (1987), and by Mack et al (1989). Here we consider the Auger electron spectra from the decay of $1 \mathrm{~s}^{2} 3 l 3 l^{\prime}$ states of $\mathrm{O}^{4+}$. These states can decay to $1 \mathrm{~s}^{2} 2 \mathrm{p}$ or to $1 \mathrm{~s}^{2} 2 \mathrm{~s}$ states of $\mathrm{O}^{5+}$ with characteristic energies given by $E_{i}-E_{2 \mathrm{p}}$ and $E_{i}-E_{2 \mathrm{~s}}$ respectively, where $E_{i}$ is the binding energy of the doubly excited state $i$, and $E_{2 \mathrm{~s}}$ and $E_{2 \mathrm{p}}$ are the energies of $1 \mathrm{~s}^{2} 2 \mathrm{~s}$ and $1 s^{2} 2 p$ states of $\mathrm{O}^{5+}$, respectively. Experimentally these states were studied in the collision of $\mathrm{O}^{6+}$ on $\mathrm{H}_{2}$ over a velocity range of 0.37 to $0.52 \mathrm{au}$. The electron spectra were measured at an angle of $50^{\circ} \pm 2^{\circ}$ with respect to the beam direction. Similar Auger spectra have been obtained by Meyer et al (1987) in the collision of $60 \mathrm{keV} \mathrm{\textrm {O } ^ { 6 + }}$ $(v=0.38 \mathrm{au})$ on $\mathrm{He}$ and on $\mathrm{H}_{2}$. In their measurements the Auger electrons were taken at $0^{\circ}$ with respect to the beam axis.

Before we compare the theoretical Auger electron energies with experiments, we first note that one needs to account for the energy shift of each Auger line due to the post-collision interaction ( $\mathrm{PCI}$ ). Since each doubly excited state of the projectile ion autoionises in the attractive field of the receding target ion, the Auger electron is slowed down and its energy is decreased by an amount, $\Delta E_{i}$, which according to the theory 
of van der Straten and Morgenstern $(1986 \mathrm{a}, \mathrm{b})$ is given by

$$
\Delta E_{i}=-\frac{Q}{2 V \tau_{i}}\left(1-\frac{V}{\left|V-v_{0}\right|}\right)
$$

where all the quantities are given in atomic units, with $Q$ the charge of the target ion, $\tau_{i}$ the lifetime of the doubly excited state, $V$ the collision velocity with speed $V$, and $\boldsymbol{v}_{0}$ the velocity of the Auger electron. To apply equation (4), one needs to know the lifetime of each doubly excited state. We use the lifetimes calculated by Vaeck and Hansen (1989) to estimate the PCI shift of both the experiments of Mack and of Meyer et al assuming $Q=2$ in equation (4).

In figure 1 we display the Auger electron spectra from these two experiments. The electron spectra predicted from our present calculation with the PCI effects included using equation (4) are also shown. Most of the Auger lines are due to the decay to $1 \mathrm{~s}^{2} 2 \mathrm{p}$ of $\mathrm{O}^{5+}$; those resulting from the decay to $1 \mathrm{~s}^{2} 2 \mathrm{~s}$ are indicated by broken lines. The latter lines are easily identified since they are shifted from the corresponding former lines by an energy difference of $E\left(1 \mathrm{~s}^{2} 2 \mathrm{p}\right)-E\left(1 \mathrm{~s}^{2} 2 \mathrm{~s}\right)=12 \mathrm{eV}$.

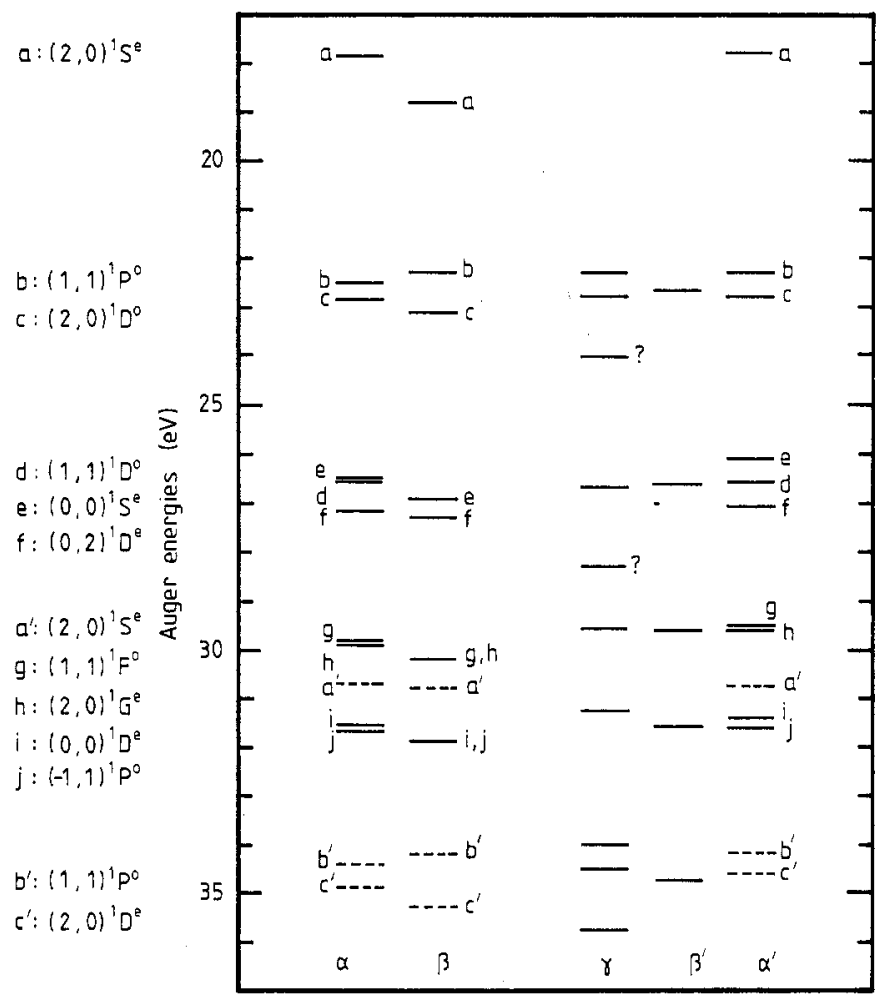

Figure 1. Comparison of $\mathrm{PCI}$-corrected theoretical energies with experimental Auger electron energies from the decay of $1 \mathrm{~s}^{2} 3 / 3 l^{\prime}$ states of $\mathrm{O}^{4+}$ to $1 \mathrm{~s}^{2} 2 \mathrm{p}$ and to $1 \mathrm{~s}^{2} 2 \mathrm{~s}$ (broken lines) of $\mathrm{O}^{5+}$. The theoretical results are from the present calculation with the PCI effect included using equation (4). The data along the column labelled $\beta$ are from Mack (1987); those along the columns $\beta^{\prime}$ and $\gamma$ are from Meyer et al (1987). The PCl-corrected theoretical results are labelled under $\alpha$ and $\alpha^{\prime}$, respectively, for the two experimental conditions (see text). 
We first compare the calculations with the results of Mack (1987). The PCI-corrected theoretical energies and the experimental results are shown along the two columns labelled $\alpha$ and $\beta$, respectively. We note that there is a reasonable matching between the calculated and the experimental energies. The results are accurate enough for identifying the specific doubly excited states populated in the collision. The major discrepancy occurs for the $(2,0){ }^{1} \mathrm{~S}^{\mathrm{e}}$ state where the error is about $0.9 \mathrm{eV}$ (the calculated $a^{\prime}$ line in the figure has been shifted by this amount). We do not show the widths of each state, but experimentally many pairs of states, such as $(b, c),(e, f),(g, h)$ and $(i, j)$, are not separated in the spectra.

We next compare the spectra of Meyer et al (1987) with the theoretical calculations. The PCI-corrected theoretical results under their experimental conditions are now shown in the column labelled $\alpha^{\prime}$. The experimental results for He target are shown in column $\gamma$, and for $\mathrm{H}_{2}$ target are shown in column $\beta^{\prime}$. We first note that for $\mathrm{He}$ target the two lines with question marks are probably triplet states resulting from double collisions. These two lines do not appear in the $\mathrm{H}_{2}$ target data, nor in the theoretical calculations for singlet states. (The lower energy line appears to be due to the $(1,1){ }^{3} \mathrm{D}^{\mathrm{e}}$ state, while the higher energy one could be due to the $(0,0){ }^{3} \mathrm{P}^{\mathrm{o}}$ state.) Double collisions may become more important for $\mathrm{He}$ target because of the smaller cross sections for double capture to $1 \mathrm{~s}^{2} 3 l 3 l^{\prime}$ states. Besides these two triplet lines, we again note that there is a reasonable correspondence between the experimental data and the calculated results. For the $\mathrm{H}_{2}$ target, the data of Mack show a pair of lines such as (b, c), which correspond to a single line in the data of Meyer et al. This results from the more elaborate fitting procedure adopted in the work of Mack to separate the overlapping lines.

The comparison in figure 1 indicates that there is a good qualitative agreement between the experiments and the calculated energies. It is not clear how accurate is the prediction of the $\mathrm{PCI}$ effect as described by equation (4). If equation (4) provides a sufficient description of the PCI effect, then measurements of the electrons at two different angles would give both the resonance energy and also the lifetime of the state. Comparison of theoretical and experimental resonance energy and width simultaneously for each line would give a more critical test of the PCI model as well as the theoretical calculations.

\subsection{Rovibrational structure of the energies of doubly excited states}

In this section we discuss the spectroscopy of $1 \mathrm{~s}^{2} 3 / 3 l^{\prime}$ states of the beryllium-like systems with that of the $3 l 3 l^{\prime}$ states of the helium-like systems. In the helium-like systems, according to the classification scheme of $\operatorname{Lin}(1983 \mathrm{a}, 1984,1986)$ the states are designated by the correlation quantum numbers $K, T$ and $A$ in addition to the usual $L, S$ and $\pi$. For states with positive and large $K$ quantum numbers, the two electrons tend to stay on opposite sides of the nucleus, while for states with negative $K$, the two electrons are on the same side of the nucleus and they have higher excitation energies (Herrick and Sinanoglu 1975, Watanabe and Lin 1986). The quantum number $T$ is the projection of $L$ onto the interelectronic axis. For the intrashell states considered here, $A \equiv+1$.

The quantum numbers $K$ and $T$, which originated from the $O(4)$ symmetry (Herrick 1975), depend on the degeneracy of hydrogen-like states when one electron is far away from the other. For beryllium-like ions, this degeneracy is removed and the basis of 
$K$ and $T$ quantum numbers no longer exists. On the other hand, the simple independent-particle model for describing each state as $3 l 3 l$ is not adequate either. To see the difference of the spectroscopy between the doubly excited states of beryllium-like and of helium-like ions, in figure 2 we display the energy levels according to the $(K, T)$ quantum numbers for $\mathrm{Be}^{2+}$ (full lines) and $\mathrm{C}^{2+}$ (broken lines). For $(K, T)=(2,0)$, we note that energies of the states ${ }^{1} \mathrm{~S}^{\mathrm{e}},{ }^{3} \mathrm{P}^{\circ},{ }^{1} \mathrm{D}^{\mathrm{e}},{ }^{3} \mathrm{~F}^{\mathrm{o}}$ and ${ }^{1} \mathrm{G}^{\mathrm{e}}$ of $\mathrm{Be}^{2+}$ can be approximated by the rotor formula, $B L(L+1)$, i.e. the spectra display the rotor structure typical of the doubly excited states of two-electron systems. This rotor structure is absent in the energies of $\mathrm{C}^{2+}$. Comparing the energies of $\mathrm{C}^{2+}$ with $\mathrm{Be}^{2+}$, we note that there is a large shift of each level in the series for states with smaller $L$, with the ${ }^{1} \mathrm{~S}^{\mathrm{e}}$ state experiencing the largest shift. This is the result of the short-range potential due to the two $1 \mathrm{~s}^{2}$ electrons in the core, which removes the degeneracy of the $3 \mathrm{~s}, 3 \mathrm{p}$ and $3 \mathrm{~d}$ states in $\mathrm{C}^{3+}$. From the $\mathrm{CI}$ viewpoint, the degree of configuration mixing is less severe for the doubly excited states of beryllium-like ions in the $3 l 3 l^{\prime}$ manifold. In fact, the lowest ${ }^{1} \mathrm{~S}^{\mathrm{e}}$ state is well represented by $3 \mathrm{~s}^{2}$ since mixture with other configurations is quite small (see table 3 below). We also note that states with large angular momentum $L$ do not shift much since these states are largely 'made' of orbitals with high $l$ values, which cannot penetrate into the core region and thus resemble pure two-electron systems.

We next point out that the spectra of doubly excited states of beryllium-like ions do not show $T$-doubling. For doubly excited states of helium-like ions, it is known that states with non-zero $T$ always have a pair of near-degenerate states that have the

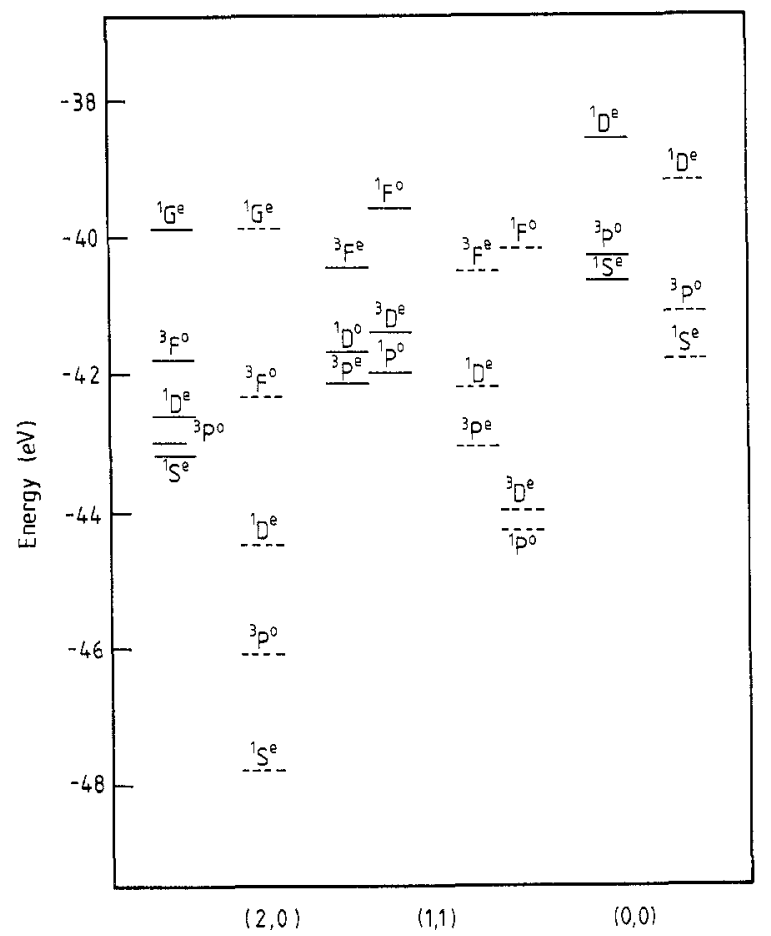

Figure 2. The 'supermultiplet' structure of the energies of the $3 / 3 l^{\prime}$ states of $\mathrm{Be}^{2+}$ and $\mathrm{C}^{2+}$. The states that have identical $(K, T)$ are grouped together vertically. Note that the rotor structure of $\mathrm{Be}^{2+}$ is absent in the spectra of $\mathrm{C}^{2+}$. 
same $L$, but opposite $\pi$ and $S$. For example, consider the $(K, T)=(1,1)$ case for $\mathrm{Be}^{2+}$ shown in figure 2. This $T$-doubling is similar to the $\Lambda$-doubling in diatomic molecules. For example, the ${ }^{3} \mathrm{P}^{\mathrm{e}}$ and ${ }^{1} \mathrm{P}^{\mathrm{o}}$ states are nearly degenerate for $\mathrm{Be}^{2+}$, with ${ }^{3} \mathrm{P}^{\mathrm{e}}$ slightly lower. These two states correspond to $3 \mathrm{p}^{2}$ and to $3 \mathrm{~s} 3 \mathrm{p}$ in the independent-particle representation. This near-degeneracy is removed in $\mathrm{C}^{2+}$. This is understood since the energy of $3 \mathrm{~s}$ is much lower than $3 \mathrm{p}$ in $\mathrm{C}^{3+}$; therefore, the $3 \mathrm{~s} 3 \mathrm{p}^{1} \mathrm{P}^{o}$ state is expected to have a lower energy than the $3 \mathrm{p}^{2}{ }^{3} \mathrm{P}^{\mathrm{e}}$ state for $\mathrm{C}^{2+}$, as shown in figure 2 .

Despite of the absence of the rotor structure and the $T$-doubling, which are characteristic of doubly excited states of two-electron atoms, in the doubly excited states of beryllium-like ions, these latter states are not represented by the independentparticle picture either. In table 3 we list the dominant $\mathrm{CI}$ coefficients for the doubly excited states of $\mathrm{Be}^{2+}$ and $\mathrm{C}^{2+}$ studied here. We note that configuration mixing is quite strong in general.

We have also calculated the energies of the $4 l 4 l^{\prime}$ states of $\mathrm{Ne}^{6+}$. The results are shown in figure 3 , where the energy levels are grouped according to the $(K, T)^{A}$ quantum numbers. By displaying the states that have the same $(K, T)^{A}$ columnwise, the energies of the states in each column increase with increasing $L$ in general. However, we do witness an irregularity. The energy of the $(1,2)^{+3} \mathrm{~F}^{\circ}$ state is slightly lower than the energy of the $(1,2)^{+1} \mathrm{D}^{\mathrm{e}}$ state, which shows that the violation of rotor structure is not only in the level spacing but also in the relative ordering with increasing $L$ along a given $(K, T)$. Only states with $L \leqslant 4$ have been calculated.

\section{Conclusions}

In this paper we investigated the energies of doubly excited states of a few beryllium-like ions. We show that by combining the configuration-interaction method with the model-potential approach the energy levels of doubly excited states can be easily calculated. We attempted to identify the Auger electron spectra of $\mathrm{O}^{4+}$ from the collision of $\mathrm{O}^{6+}$ with $\mathrm{H}_{2}$ and with $\mathrm{He}$. By including the effect of post-collision interaction, it is possible to identify the doubly excited states populated in the collisions. There are still quantitative discrepancies between the calculated results and experiments. It is suggested that measurements of Auger electrons be carried out at least at two angles to test the present model of the PCI effect. Such measurements would allow the comparison of the theoretical and experimental energy position and width simultaneously for each Auger line.

We have also addressed the systematics of the energy levels of the doubly excited states of beryllium-like ions and compare it with those of helium-like ions. We note that the rotor structure and the $T$-doubling that are characteristic of helium-like doubly excited states are absent for beryllium-like ions, but the relative energy ordering according to the $(K, T)^{A}$ scheme still holds in general. This further supports the view that the $(K, T)^{A}$ scheme is preferable to the independent-particle representation for designating doubly excited states.

\section{Acknowledgments}

This work was supported in part by the US Department of Energy, Office of Basic Energy Sciences, Division of Chemical Sciences. We thank R Morgenstern for clarifying several points about the $\mathrm{PCI}$ corrections and $\mathrm{N}$ Stolterfoht and $\mathrm{R}$ Morgenstern for 
Table 3. Comparison of the dominant $\mathrm{Cl}$ coefficients for $\mathrm{Be}^{2+}$ and $\mathrm{C}^{2+}$.

\begin{tabular}{|c|c|c|c|}
\hline & & $\mathrm{Be}^{2+}$ & $\mathrm{C}^{2+}$ \\
\hline \multirow[t]{3}{*}{$(2,0){ }^{1} \mathrm{~S}^{\mathrm{e}}$} & $3 \mathrm{~s} 3 \mathrm{~s}$ & 0.760 & 0.932 \\
\hline & $3 p 3 p$ & 0.620 & 0.332 \\
\hline & $3 \mathrm{~d} 3 \mathrm{~d}$ & 0.144 & - \\
\hline \multirow[t]{4}{*}{$(0,0){ }^{1} \mathrm{~S}^{\mathrm{e}}$} & $3 \mathrm{~s} 3 \mathrm{~s}$ & -0.524 & -0.253 \\
\hline & $3 p 3 p$ & 0.477 & 0.753 \\
\hline & $3 \mathrm{~d} 3 \mathrm{~d}$ & 0.657 & 0.568 \\
\hline & $3 d 4 d$ & -0.218 & -0.178 \\
\hline \multirow[t]{6}{*}{$(-2,0){ }^{1} S^{e}$} & $3 \mathrm{~s} 3 \mathrm{~s}$ & 0.330 & 0.183 \\
\hline & $3 p 3 p$ & -0.549 & -0.500 \\
\hline & $3 \mathrm{~d} 3 \mathrm{~d}$ & 0.651 & 0.729 \\
\hline & $3 p 4 p$ & 0.256 & 0.315 \\
\hline & $3 s 4 s$ & -0.186 & 0.093 \\
\hline & $3 \mathrm{~d} 4 \mathrm{~d}$ & -0.146 & -0.188 \\
\hline \multirow[t]{3}{*}{$(1,1)^{1} \mathrm{P}^{o}$} & $3 \mathrm{~s} 3 \mathrm{p}$ & 0.737 & 0.875 \\
\hline & $3 p 3 d$ & 0.646 & 0.457 \\
\hline & $3 p 4 d$ & -0.115 & -0.090 \\
\hline \multirow[t]{6}{*}{$(-1,1)^{1} \mathrm{P}^{\circ}$} & $3 s 3 p$ & -0.616 & -0.419 \\
\hline & $3 p 3 d$ & 0.683 & 0.825 \\
\hline & $3 s 4 p$ & 0.114 & 0.190 \\
\hline & $3 \mathrm{p} 4 \mathrm{~s}$ & 0.161 & - \\
\hline & $3 \mathrm{~d} 4 \mathrm{p}$ & -0.155 & -0.147 \\
\hline & $3 p 4 f$ & -0.274 & -0.258 \\
\hline \multirow[t]{2}{*}{$(2,0)^{3} \mathrm{P}^{\circ}$} & $3 s 3 p$ & 0.913 & 0.971 \\
\hline & $3 p 3 d$ & 0.383 & 0.201 \\
\hline \multirow[t]{5}{*}{$(0,0){ }^{3} \mathrm{P}^{\circ}$} & $3 s 3 p$ & -0.381 & -0.197 \\
\hline & $3 p 3 d$ & 0.892 & 0.952 \\
\hline & $3 p 4 d$ & -0.107 & -0.118 \\
\hline & $3 \mathrm{~d} 4 \mathrm{p}$ & 0.128 & 0.100 \\
\hline & $3 \mathrm{~d} 4 \mathrm{f}$ & -0.125 & -0.122 \\
\hline \multirow[t]{4}{*}{$(2,0){ }^{1} \mathrm{D}^{\mathrm{e}}$} & $3 \mathrm{~s} 3 \mathrm{~d}$ & -0.616 & -0.789 \\
\hline & $3 p 3 p$ & 0.754 & 0.579 \\
\hline & $3 p 4 p$ & -0.101 & - \\
\hline & $3 \mathrm{~d} 3 \mathrm{~d}$ & 0.149 & - \\
\hline \multirow[t]{5}{*}{$(0,2){ }^{1} \mathrm{D}^{\mathrm{e}}$} & $3 \mathrm{~s} 3 \mathrm{~d}$ & 0.550 & 0.474 \\
\hline & $3 p 3 p$ & 0.300 & 0.654 \\
\hline & $3 \mathrm{~d} 3 \mathrm{~d}$ & 0.746 & 0.553 \\
\hline & $3 d 4 d$ & 0.181 & -0.132 \\
\hline & $3 p 4 f$ & - & -0.104 \\
\hline \multirow[t]{7}{*}{$(0,0){ }^{1} D^{e}$} & $3 \mathrm{~s} 3 \mathrm{~d}$ & -0.492 & -0.286 \\
\hline & $3 p 3 p$ & -0.528 & -0.439 \\
\hline & $3 \mathrm{~d} 3 \mathrm{~d}$ & 0.588 & 0.786 \\
\hline & $3 p 4 p$ & 0.155 & 0.123 \\
\hline & $3 p 4 f$ & 0.249 & 0.218 \\
\hline & $3 \mathrm{~d} 4 \mathrm{~s}$ & 0.133 & - \\
\hline & $3 \mathrm{~d} 4 \mathrm{~d}$ & -0.115 & -0.169 \\
\hline \multirow[t]{4}{*}{$(1,1){ }^{3} \mathrm{D}^{\mathrm{e}}$} & $3 \mathrm{~s} 3 \mathrm{~d}$ & 0.977 & 0.982 \\
\hline & $3 \mathrm{~s} 4 \mathrm{~d}$ & -0.105 & -0.119 \\
\hline & $4 p 4 f$ & -0.113 & -0.101 \\
\hline & $3 \mathrm{~d} 4 \mathrm{~s}$ & 0.122 & - \\
\hline
\end{tabular}


Table 3. (continued)

\begin{tabular}{rrr}
\hline & $\mathrm{Be}^{2+}$ & \multicolumn{1}{c}{$\mathrm{C}^{2+}$} \\
\hline$(1,1)^{1} \mathrm{~F}^{\circ} 3 \mathrm{p} 3 \mathrm{~d}$ & 0.941 & 0.932 \\
$3 \mathrm{~s} 4 \mathrm{f}$ & 0.196 & 0.276 \\
$3 \mathrm{~d} 4 \mathrm{p}$ & -0.196 & -0.140 \\
$3 \mathrm{~d} 4 \mathrm{f}$ & -0.142 & -0.128 \\
$(2,0){ }^{3} \mathrm{~F}^{\circ} 3 \mathrm{p} 3 \mathrm{~d}$ & 0.978 & 0.980 \\
$3 \mathrm{~s} 4 \mathrm{f}$ & - & 0.105 \\
$3 \mathrm{p} 4 \mathrm{~d}$ & -0.137 & -0.137 \\
$(2,0){ }^{1} \mathrm{G}^{\mathrm{e}} 3 \mathrm{~d} 3 \mathrm{~d}$ & 0.944 & 0.945 \\
$3 \mathrm{~d} 4 \mathrm{~d}$ & -0.237 & -0.233 \\
$3 \mathrm{p} 4 \mathrm{f}$ & 0.201 & 0.212 \\
\hline
\end{tabular}

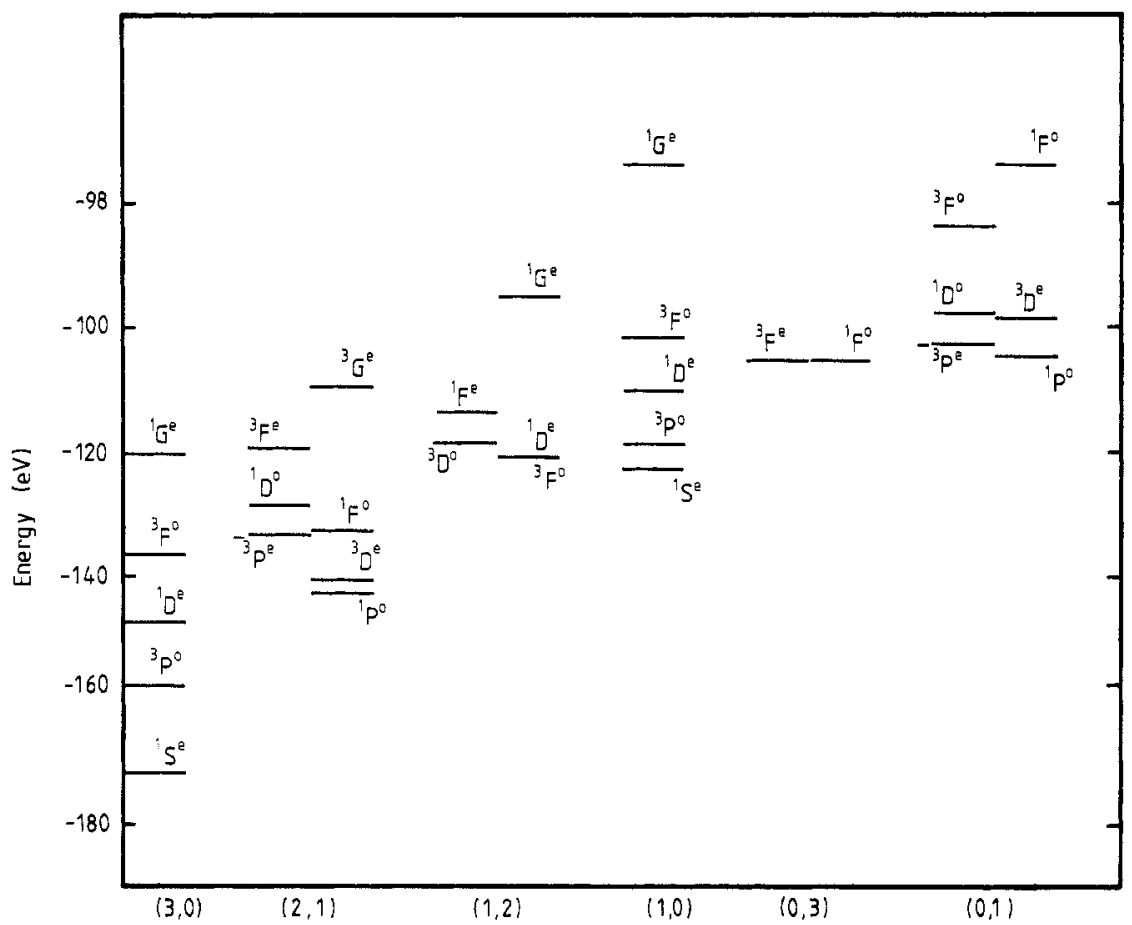

Figure 3. Energy levels of $1 \mathrm{~s}^{2} 4 l 4 l^{\prime}$ states of $\mathrm{Ne}^{6+}$ grouped in the form of 'supermultiplet' structure. The binding energies are given in electronvolts.

discussing the various aspects of the experimental electron spectra. We also thank $\mathrm{J}$ Hansen for communicating their results prior to publications.

Note added in proof. After the completion of this work, we received the preprint of Vaeck and Hansen where the energy levels, radiative and autoionisation widths of the states studied in this paper were calculated in a different $\mathrm{Cl}$ method. We use their calculated lifetimes in the analysis of the PCI effects in the revised version. 


\section{References}

Bordenave-Montesquieu A, Benoit-Cattin P, Boudjema P, Gleizes A and Bachau H 1987 J. Phys. B: At. Mol. Phys. 20 L695-703

Christensen-Dalsgaard B L 1985 J. Phys. B: At. Mol. Phys. 18 L407-11

Fritsch W and Lin C D 1986 J. Phys. B: At. Mol. Phys. 19 2683-94

Greene C H 1981 Phys. Rev. A 23 661-78

Herrick D R 1975 Phys. Rev. A 12 413-24

Herrick D R and Sinanoglu O 1975 Phys. Rev. A 11 97-110

Ho Y K 1979 J. Phys. B: At. Mol. Phys. $12387-99$ 1987 Phys. Rev. A 35 2035-43

Laughlin C and Victor G A 1972 Atomic Physics vol 3, ed S J Smith and G K Walters (New York: Plenum) Lin C D 1983a Phys. Rev. Lett. 51 1348-51

— 1983b J. Phys. B: At. Mol. Phys. 16 723-36

- 1984 Phys. Rev. A 29 1019-33

1986 Adv. At. Mol. Phys. 22 77-142

1989 Phys. Rev. A 39 4355-61

Lipsky L, Anaia R and Conneely M J 1977 At. Data Nucl. Data Tables 20 127-41

Mack R 1987 PhD Thesis Utrecht University (unpublished)

Mack M, Nijland J H, van der Straten P, Niehaus A and Morgenstern R 1989 Phys. Rev. A 39 3846-54

Mann R 1987 Phys. Rev. A 35 4988-5004

Martín F, Mó O, Riera A and Yáñez M 1988 Phys. Rev. A 38 1094-7

Meyer F W, Griffin D C, Havener C C, Hug M S, Phaneuf R A, Swensen J K and Stolterfoht N 1987 Proc. 15th Int. Conf. on Physics of Electronic and Atomic Collisions, Brighton ed H B Gilbody et al (Amsterdam: North-Holland)

Moore C E 1949 Atomic Energy Levels NBS Circular No 467, vol 1 (Washington, DC: US Govt Printing Office)

Moore D L and Norcross D W 1974 Phys. Rev. A 10 1646-57

Norcross D W and Seaton M J 1969 J. Phys. B: At. Mol. Phys, 2 731-40

Oza D H 1987 J. Phys. B: At. Mol. Phys. 20 L13-7.

Stewart R F, Laughlin C and Victor G A 1974 Chem. Phys, Lett. 29 353-8

Vaeck N and Hansen J E 1989 J. Phys. B: At. Mol. Phys. 22 in press

Van der Straten P and Morgenstern R 1986a J. Phys. B: At. Mol. Phys. 19 1361-70

- 1986b Phys. Rev. A 34 4482-4

Watanabe S and Lin C D 1986 Phys. Rev. A 34 823-37 